\title{
A New Frequency Domain System Identification Method
}

\author{
Alexander Stotsky* \\ * Signals \& Systems, Chalmers University of Technology, Gothenburg, \\ SE-41296, Sweden (e-mail: alexander.stotsky@chalmers.se)
}

\begin{abstract}
A new frequency domain system identification method based on a multi-frequency input signal is proposed. Frequency contents of the oscillating signal are estimated using a modified Kaczmarz algorithm proposed in this paper. Lyapunov stability analysis is performed for this new Kaczmarz algorithm and transient bounds for estimation error are established. Moreover, a new method for estimation of the variance of the measurement noise in Kaczmarz algorithms is also described. A comparison of a transient performance of modified Kaczmarz algorithm and a recursive least-squares algorithm is presented. The results are applied to a frequency domain identification of a DC motor.
\end{abstract}

\section{INTRODUCTION}

A property of a linear time-invariant system to be completely characterized by the steady-state response to harmonic input is the basis for the frequency domain system identification methods [9]. A harmonic input (a cosine wave) of a certain frequency applied to linear system induces oscillations of the output of the same frequency with different magnitude and phase after some transient. The magnitude of the output signal is equal to the magnitude of the input signal multiplied by the module of the transfer function, and the phase shift is equal to the argument of the transfer function.

The frequency response of system can be measured by applying a test harmonic signal of a certain frequency and measuring magnitude and phase of the output signal at that frequency. This procedure is repeated for a number of frequencies in a specified frequency range and only one frequency at a time is used.

This method is time consuming since the response is measured sequentially (only one frequency at a time is used) for a number of frequencies. The identification method proposed in this paper applies a multi-frequency harmonic signal that contains a number of distinct frequencies, and the response is measured simultaneously at each frequency, recovering magnitude and phase. This in turn, essentially reduces a costly time of experiment, providing the same accuracy of estimation.

The model for the output signal is specified in a form of a trigonometric polynomial (a sum of sines and cosines of known frequencies specified in the input signal) with adjustable coefficients.

Many algorithms can be applied for estimation of the frequency contents of oscillating signals. One of such algorithms is a simple Kaczmarz algorithm [1] where the output of the model matches the measured signal exactly in each discrete step and the vector of the parameter mismatch is orthogonal to the regressor vector. Kaczmarz algorithm was successfully tested for estimation of the frequency contents of the high resolution engine speed signal [11] in automotive applications. A computational simplicity and robustness against a measurement noise make Kaczmarz algorithm very attractive for estimation of the frequency contents of complex signals with a large number of frequencies in real-time applications. Kaczmarz algorithm however, suffers from the following drawbacks: it neither allows the estimation of the variance of measurement noise nor selection of the order a trigonometric polynomial in the case where new frequencies appear in the signal. A gain update algorithm with a forgetting factor is introduced in this paper aiming to the performance improvement of a Kaczmarz projection method. Stability analysis is performed for modified algorithm in this paper unlike previous publications where similar modifications of Kaczmarz algorithm were reported [2],[11].

The performance of modified Kaczmarz algorithm is compared in this paper to the performance of Recursive LeastSquares (RLS) algorithm. Modified algorithm is applied to the frequency domain identification of DC motor.

Notice that the idea of using broadband excitation signals such as multi-sine signals for a frequency domain system identification was also discussed in [14] (see also references therein). The output of the system which is excited by a broadband signal is analyzed via a standard Fast Fourier Transform (FFT) method that limits the frequencies of the input signal to the set of Fourier frequencies i.e., a fundamental frequency accompanied by higher-frequency harmonics. Any distinct frequencies of the input signal can be used in the frequency domain system identification method described in this paper.

Contributions of this paper can be summarized as follows: 1) a new frequency domain system identification method based on a multi-frequency input signal applied to a frequency domain identification of DC motor ; 2) a new Kaczmarz algorithm with a least-squares gain update for estimation of the frequency contents of the oscillating signals; 3) Lyapunov stability analysis for a new Kaczmarz algorithm with a least-squares gain update and transient bounds for estimation error ; 4) a new method for estimation of the variance of the measurement noise in Kaczmarz algorithms ; 5) a comparison of performance of modified Kaczmarz algorithm and a recursive least-squares 
algorithm.

This paper is organized as follows. A new frequency domain system identification method is proposed in the next Section 2. A new modified Kaczmarz algorithm is introduced in Section 3 and applied to a frequency domain identification of DC motor in Section 4. The paper is concluded by a discussion presented in Section 5 .

\section{A NEW FREQUENCY DOMAIN IDENTIFICATION METHOD BASED ON MULTI-FREQUENCY INPUT SIGNAL}

A harmonic input $u_{k}$ of a certain frequency $q$ applied to a linear system with the frequency transfer function $H(i q)$, where $i^{2}=-1$ induces oscillations of the output $y_{k}$ of the same frequency $q$ with different magnitude and phase after some transient. The magnitude of the output signal is equal to the magnitude of the input signal multiplied by the module of the transfer function, and the phase shift is equal to the argument of the transfer function.

Traditional method of the frequency response measurements is time consuming since the response is measured sequentially for a number of frequencies and only one frequency at a time is used. The identification method described in this Section applies the composite harmonic signal to the input that contains the number of distinct frequencies, and the response is measured simultaneously at each frequency, recovering the magnitude and the phase. The multi-frequency test input sequence $u_{k}$ is chosen as follows:

$$
u_{k}=\sum_{q=q_{1}}^{q_{n}} A_{q} \cos (q k \Delta)
$$

where $A_{q}$ is the amplitude of harmonic signal at a frequency $q=q_{1}, \ldots, q_{n}, n$ is the number of frequencies involved, $k=0,1,2, \ldots$.

The principle of superposition implies that a sum of cosine waves as an input forces cosine waves at the output with the same frequencies after a transient and therefore measurements of the output result in the following sequence:

$$
y_{k}=\sum_{q=q_{1}}^{q_{n}} B_{q *} \cos \left(q k \Delta+\psi_{q *}\right)+\xi_{k}
$$

where magnitude of the response is $B_{q *}=|H(i q)| A_{q}$, and the phase $\psi_{q *}=\arg (H(i q))$ are treated as uncertain parameters, where $|H(i q)|=\frac{B_{q *}}{A_{q}}$ is the module/magnitude and $\arg (H(i q))$ is the argument of the transfer function evaluated at a frequency $q, \xi_{k}$ is a zero mean white Gaussian measurement noise with a variance $\sigma^{2}$.

Output sequence (2) can be rewritten as follows:

$$
y_{k}=\sum_{q=q_{1}}^{q_{n}} a_{q *} \cos (q k \Delta)+b_{q *} \sin (q k \Delta)+\xi_{k}
$$

where the relation between equations (2) and (3) is given by magnitude $B_{q *}=\sqrt{a_{q *}^{2}+b_{q *}^{2}}$ and phase $\psi_{q *}=$ $-\arctan \frac{b_{q *}}{a_{q *}}$, where $a_{q *}$ and $b_{q *}$ are unknown coefficients of the output trigonometric polynomial.
Finally, the output sequence can be presented in the following vector form:

$$
y_{k}=\varphi_{k}^{T} \theta_{*}+\xi_{k}
$$

where $\varphi_{k}$ is the regressor and $\theta_{*}$ is the vector of constant unknown parameters defined as follows:

$$
\begin{aligned}
& \varphi_{k}^{T}=\left[\cos \left(q_{1} k \Delta\right) \sin \left(q_{1} k \Delta\right) \cos \left(q_{2} k \Delta\right)\right. \\
& \left.\sin \left(q_{2} k \Delta\right) \ldots \cos \left(q_{n} k \Delta\right) \sin \left(q_{n} k \Delta\right)\right] \\
& \theta_{*}^{T}=\left[\begin{array}{lllllll}
a_{q 1 *} & b_{q 1 *} & a_{q 2 *} & b_{q 2 *} & \ldots & a_{q n *} & b_{q n *}
\end{array}\right]
\end{aligned}
$$

In other words the output sequence is presented in a form of trigonometric polynomial with known frequencies and unknown coefficients.

The model of the output sequence can be introduced as follows:

$$
\hat{y}_{k}=\varphi_{k}^{T} \theta_{k}
$$

where $\varphi_{k}$ is the regressor defined in (5) and $\theta_{k}$ is the following vector of adjustable parameters:

$$
\theta_{k}^{T}=\left[\begin{array}{lllllll}
a_{q 1 k} & b_{q 1 k} & a_{q 2 k} & b_{q 2 k} & \ldots & a_{q n k} & b_{q n k}
\end{array}\right]
$$

A parameter estimation aim is to find an update law for the parameter vector $\theta_{k}$ such that $\theta_{k}$ converges to $\theta_{*}$ i.e., the following holds:

$$
E\left[\theta_{k}-\theta_{*}\right]=0
$$

at steady-state, provided that the regressor $\varphi_{k}$ is persistently exciting i.e., there exist positive constants $\rho_{0}, \rho_{1}$ and $w$ such that the following inequality holds:

$$
0<\rho_{0} I \leq \sum_{p=k}^{p=k+w} \varphi_{p} \varphi_{p}^{T} \leq \rho_{1} I
$$

where $E$ is a mathematical expectation and $I$ is the identity matrix. Inequalities (10) are valid for any distinct frequencies of the input sequence provided that a zero division in the elements of the information matrix $\sum_{p=k}^{p=k+w} \varphi_{p} \varphi_{p}^{T}$ is avoided [11].

A frequency domain system identification aim is the convergence of the magnitude and phase:

$$
\begin{aligned}
E\left[B_{q k}-B_{q *}\right] & =0 \\
E\left[\psi_{q k}-\psi_{q *}\right] & =0
\end{aligned}
$$

where

$$
\begin{aligned}
& B_{q k}=\sqrt{a_{q k}^{2}+b_{q k}^{2}} \\
& \psi_{q k}=-\arctan \frac{b_{q k}}{a_{q k}}
\end{aligned}
$$

and $a_{q k}$ and $b_{q k}$ are adjustable parameters defined in (8), $q=q_{1}, \ldots, q_{n}$ and $|\hat{H}(i q)|=\frac{B_{q k}}{A_{q}}$.

The achievement of the system identification aim (11),(12) follows from the achievement of the parameter estimation aim (9) as can be shown using linearization technique. Hence the system identification problem is reduced to the problem of estimation of the coefficients of trigonometric 
polynomial.

Many algorithms can be applied to solve the parameter estimation problem (see for example algorithms described in $[3],[4],[7],[9])$, where the parameter convergence is granted by the condition of persistency of excitation (10). The faster the parameter convergence the less time is required for a system identification, gaining the advantage of proposed approach with respect to a conventional one. Therefore the priority in this application should be given to the fast convergent algorithms leading to a rapid frequency domain system identification method.

\section{A NEW MODIFIED KACZMARZ ALGORITHM}

\subsection{A Least-Squares Gain Update with Forgetting Factor}

Lemma 1. Consider the following gain update law

$$
\Gamma_{k}^{-1}=\lambda_{0} \Gamma_{k-1}^{-1}+\lambda_{1} \varphi_{k} \varphi_{k}^{T}, \quad \Gamma_{0}^{-1}=\gamma_{0} I, \quad \gamma_{0}>0,
$$

where $k=1,2,3 \ldots$ and $0<\lambda_{0}<1$ is a forgetting factor and $\lambda_{1}$ is a gain factor with regressor vector (5). Then there exist a positive constant $\lambda_{0 *}$ sufficiently close to one such that for all $\lambda_{0}$ from the interval $\lambda_{0 *}<\lambda_{0}<1$, and for a sufficiently large $\gamma_{0}>0$ the matrix $\Gamma_{k}^{-1}, k=0,1,2, \ldots$ is an SDD matrix ${ }^{1}$ i.e., the following inequalities are valid

$$
\left|\gamma_{i i}\right|>\sum_{j=1, j \neq i}^{2 n+1}\left|\gamma_{i j}\right| \quad i=1, \ldots,(2 n+1)
$$

for all admissible frequencies, where $\gamma_{i j}, i, j=1, \ldots,(2 n+$ 1) are the elements of matrix $\Gamma_{k}^{-1}, \gamma_{i i}$ are positive diagonal elements of matrix $\Gamma_{k}^{-1}$ and $n$ is the number of frequencies in signal (4).

Moreover, the matrix $\Gamma_{k}^{-1}$ is a positive definite matrix, it has positive eigenvalues only for $k=0,1,2, \ldots$ and the following inequalities are true:

$$
0<\lambda_{\min }\left(\Gamma_{k}^{-1}\right) I \leq \Gamma_{k}^{-1} \leq \lambda_{\max }\left(\Gamma_{k}^{-1}\right) I
$$

where $\lambda_{\min }\left(\Gamma_{k}^{-1}\right)>0$ and $\lambda_{\max }\left(\Gamma_{k}^{-1}\right)>0$ are minimal and maximal eigenvalues of the matrix $\Gamma_{k}^{-1}$ respectively, and $I$ is the identity matrix.

Finally, the following bounds are valid for minimal and maximal eigenvalues of the matrix $\Gamma_{k}^{-1}$ :

$$
\begin{aligned}
& \lambda_{\min }\left(\Gamma_{k}^{-1}\right) \geq \min _{i}\left\{\gamma_{i i}-\sum_{j=1, j \neq i}^{2 n+1}\left|\gamma_{i j}\right|\right\}>0 \\
& \lambda_{\max }\left(\Gamma_{k}^{-1}\right) \leq \max _{i}\left\{\gamma_{i i}+\sum_{j=1, j \neq i}^{2 n+1}\left|\gamma_{i j}\right|\right\}
\end{aligned}
$$

for $i=1, \ldots,(2 n+1)$.

Lemma 1 is proved via explicit evaluation of the elements of matrix $\Gamma_{k}^{-1}$. This evaluation is performed in [13].

Remark 1. Here and below extremely low frequencies $q$ for which $\cos (q)$ is very close to one are not included in the set of admissible frequencies.

1 A matrix is said to be a strictly diagonally dominant (SDD) matrix if in every row of the matrix, the magnitude of the diagonal entry in that row is larger than the sum of the magnitudes of all the other (non-diagonal) entries in that row [6]
A Kaczmarz update law [1] is modified in the next Subsection with a least-squares gain update described above aiming to the performance improvement. The properties of matrix $\Gamma_{k}^{-1}$ reported in Lemma 1 are very useful in stability analysis performed in Subsection 3.2 where the matrix $\Gamma_{k}^{-1}$ is used in a Lyapunov function candidate.

\subsection{Kaczmarz Projection Algorithm with the Gain Update}

Lemma 2. Consider the system (4), (7) with bounded measurement noise $\left|\xi_{k}\right| \leq c_{\xi}, c_{\xi}>0$ and the following update law for $\theta_{k}$ :

$$
\begin{aligned}
\theta_{k} & =\theta_{k-1}+\frac{\Gamma_{k-1} \varphi_{k}}{\varphi_{k}^{T} \Gamma_{k-1} \varphi_{k}}\left(y_{k}-\theta_{k-1}^{T} \varphi_{k}\right) \\
\Gamma_{k} & =\frac{1}{\lambda_{0}}\left[\Gamma_{k-1}-\frac{\Gamma_{k-1} \varphi_{k} \varphi_{k}^{T} \Gamma_{k-1}}{\left(\frac{\lambda_{0}}{\lambda_{1}}+\varphi_{k}^{T} \Gamma_{k-1} \varphi_{k}\right)}\right] \\
\Gamma_{0} & =\frac{1}{\gamma_{0}} I, \quad \gamma_{0}>0, \frac{1}{2}<\lambda_{0}<1, \lambda_{1}>0 .
\end{aligned}
$$

Then the following transient bound is valid for estimation error $\tilde{\theta}_{k}=\theta_{k}-\theta_{*}$ :

$$
\begin{aligned}
& \left\|\tilde{\theta}_{k}\right\| \leq \sqrt{\frac{\lambda_{0}^{k} V_{0}+b \frac{\left(1-\lambda_{0}^{k}\right)}{\left(1-\lambda_{0}\right)}}{\lambda_{\min }\left(\Gamma_{k}^{-1}\right)}}, \\
& b=\left[\lambda_{1}+\frac{\lambda_{u}}{(n+1)} \frac{\left(1-\lambda_{0}\right)^{2}}{\left(2 \lambda_{0}-1\right)}\right. \\
& \left.+\frac{\lambda_{u}}{n+1}\right] c_{\xi}^{2}
\end{aligned}
$$

where $\lambda_{\min }\left(\Gamma_{k}^{-1}\right)$ is a minimal eigenvalue of the matrix $\Gamma_{k}^{-1}$ in step $k=0,1,2, \ldots$ and $\lambda_{u}>0$ is an upper bound for $\lambda_{\max }\left(\Gamma_{k}^{-1}\right)$ i.e., $\lambda_{\max }\left(\Gamma_{k}^{-1}\right) \leq \lambda_{u}, V_{0}=\tilde{\theta}_{0}^{T} \Gamma_{0}^{-1} \tilde{\theta}_{0}, k=$ $0,1,2, \ldots$

The proof of Lemma 2 is given in [13] and is based on the Lyapunov function $V_{k}=\tilde{\theta}_{k}^{T} \Gamma_{k}^{-1} \tilde{\theta}_{k}$ applied to the following error model:

$$
\begin{aligned}
\varphi_{k}^{T} \tilde{\theta}_{k} & =\xi_{k} \\
\tilde{\theta}_{k}-\tilde{\theta}_{k-1} & =-\frac{\Gamma_{k-1} \varphi_{k} \varphi_{k}^{T} \tilde{\theta}_{k-1}}{\varphi_{k}^{T} \Gamma_{k-1} \varphi_{k}}+\frac{\Gamma_{k-1} \varphi_{k}}{\varphi_{k}^{T} \Gamma_{k-1} \varphi_{k}} \xi_{k} \\
\Gamma_{k}^{-1} & =\lambda_{0} \Gamma_{k-1}^{-1}+\lambda_{1} \varphi_{k} \varphi_{k}^{T}
\end{aligned}
$$

which is valid for system (4), (7) and (20),(21).

Corollary. In the noise-free case, where $\xi_{k}=0$ and $c_{\xi}=0$ the following transient bound is valid:

$$
\left\|\tilde{\theta}_{k}\right\| \leq \sqrt{\frac{\lambda_{0}^{k} V_{0}}{\lambda_{\min }\left(\Gamma_{k}^{-1}\right)}}
$$

Remark 2. For the case where $\lambda_{0}=1$ the minimal eigenvalue of the matrix $\Gamma_{k}^{-1}$ tends to infinity i.e., $\lambda_{\min }\left(\Gamma_{k}^{-1}\right) \rightarrow$ $\infty$ whereas $\lambda_{\min }\left(\Gamma_{k}^{-1}\right)$ remain bounded for $\lambda_{0}<1$. Therefore $\left\|\tilde{\theta}_{k}\right\| \rightarrow 0$ as $k \rightarrow \infty$ in both cases due to (27). 


\subsection{Estimation of a Variance of Measurement Noise and an Order of Trigonometric Polynomial}

The method for estimation of the variance of the measurement noise $\sigma^{2}$ is based on relation (24) which relates the regressor vector, the vector of the parameter mismatch and measurement noise. The variance can be calculated due the fact that the algorithm is unbiased i.e., a mathematical expectation of parameter vector is equal to a vector of true parameters at steady-state [12].

Squaring both sides of (24) and taking mathematical expectation yields:

$$
E\left[\tilde{\theta}_{k}^{T} \varphi_{k} \varphi_{k}^{T} \tilde{\theta}_{k}\right]=E\left[\xi^{2}\right]=\sigma^{2}, \quad \tilde{\theta}_{k}=\theta_{k}-\theta_{*}
$$

The relation above gives the following estimate of the variance of the measurement noise:

$$
\hat{\sigma}^{2}=\frac{1}{N} \sum_{k=1}^{N}\left(\theta_{k}-\bar{\theta}\right)^{T} \varphi_{k} \varphi_{k}^{T}\left(\theta_{k}-\bar{\theta}\right)
$$

where $\bar{\theta}=\frac{1}{N} \sum_{k=1}^{N} \theta_{k}$ is an average value of the parameter vector $\theta_{k}$ at steady-state. The right-hand side of (29) is an estimate of mathematical expectation $E\left[\tilde{\theta}_{k}^{T} \varphi_{k} \varphi_{k}^{T} \tilde{\theta}_{k}\right]$ where the vector of unknown parameters $\theta_{*}$ is replaced by the average value $\bar{\theta} \quad\left(E\left[\theta_{k}\right]=\theta_{*}\right)$ for a sufficiently large sample size $N$ at steady-state.

This variance estimation technique (29) is based on estimation of a mathematical expectation which is well-estimated via a sample average for a sufficiently large sample size only. A histogram of an estimate of variance (29) plotted in Figure 1 shows a pinpoint accuracy of estimation for a sufficiently large size.

Equation (29) is a useful tool for estimation of the order of trigonometric polynomial (4). The order of the polynomial should be increased (new frequencies should be added to a model) until estimate (29) of the variance of the measurement noise is reduced and this reduction is statistically significant.

\subsection{Comparative Analysis of Modified Kaczmarz Algorithm and Recursive Least-Squares Algorithm}

Modified Kaczmarz algorithm (20),(21) proposed in this paper is similar to Recursive Least Squares (RLS) with a forgetting factor algorithm which can be written as follows $[9]$ :

$$
\begin{aligned}
\theta_{k} & =\theta_{k-1}+\frac{\Gamma_{k-1} \varphi_{k}}{\lambda_{0}+\varphi_{k}^{T} \Gamma_{k-1} \varphi_{k}}\left(y_{k}-\theta_{k-1}^{T} \varphi_{k}\right) \\
\Gamma_{k} & =\frac{1}{\lambda_{0}}\left[\Gamma_{k-1}-\frac{\Gamma_{k-1} \varphi_{k} \varphi_{k}^{T} \Gamma_{k-1}}{\left(\lambda_{0}+\varphi_{k}^{T} \Gamma_{k-1} \varphi_{k}\right)}\right]
\end{aligned}
$$

where $0<\lambda_{0} \leq 1$ is a forgetting factor. The matrix $\Gamma_{k}$ is initialized to the inverse of information matrix after several initial steps and then updated recursively using (31).

Modified Kaczmarz algorithm (20),(21) and RLS algorithm $(30),(31)$ look very similar, but the matrix $\Gamma_{k}$ plays different role in these algorithms. This matrix is a gain matrix in modified Kaczmarz algorithm (20),(21) whereas the matrix $\Gamma_{k}$ is a recursive estimate of the inverse of the information matrix in RLS algorithm (30),(31). The

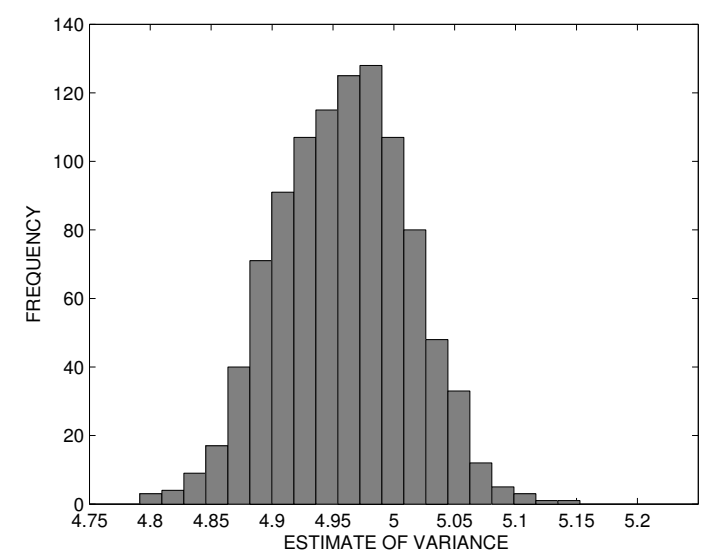

FIG 1. A histogram of an estimate of variance $\hat{\sigma}^{2}$. The estimate of the variance $\hat{\sigma}^{2}$ is calculated using (29) with a sample size of $N=17000$. The number of data points shown in histogram is equal to one thousand, distributed over twenty bins. System (4),(20),(21) with a true variance $\sigma^{2}=5$ was simulated. An average value of the sample variance $\hat{\sigma}^{2}$ is equal to 4.9593 and a sample standard deviation is equal to 0.0539 .

inverse of the information matrix can be calculated when this matrix gets a full rank. Modified Kaczmarz algorithm (20),(21) provides estimates which are close to correct values even at the initial steps whereas RLS algorithm $(30),(31)$ has a delay time until information matrix becomes invertible.

Robustness of these two algorithms against accumulation errors can be compared by adding an equal perturbation term $0.26 I$, where $I$ is a unity matrix, to the right hand side of equations for the matrix $\Gamma_{k}$ in (21) and (31). Estimates provided by RLS algorithm oscillate whereas a modified Kaczmarz algorithm exhibits a robust behavior and even a satisfactory performance as it is shown in Figure 2. As it was mentioned above the matrix $\Gamma_{k}$ in RLS algorithm is a recursive estimate of the inverse of information matrix and errors in $\Gamma_{k}$ destroy optimality and even might have impact on stability of the algorithm. On the contrary the matrix $\Gamma_{k}$ in modified Kaczmarz algorithm is treated just as a gain matrix which appears to be more tolerant to the error accumulation.

Finally, three following algorithms: Kaczmarz algorithm (20) with $\Gamma_{k}=I$, modified Kaczmarz algorithm (20),(21), and RLS algorithm (30),(31) are compared in terms of the variance of the parameter error $E\left\|\tilde{\theta}_{k}\right\|^{2}$ in Figure 3. RLS algorithm shows better performance for forgetting factors which are close to one. However, modified Kaczmarz algorithm is more robust with respect to accumulation errors and provide estimates which are close to the true values even at the initial steps of the algorithm. It can also be shown that modified Kaczmarz algorithm converges much faster than classical Kaczmarz algorithm. Therefore modified algorithm is applied in the next Section to a frequency domain identification of DC motor.

Notice that the comparative analysis presented above is valid for a persistently exciting regressor only and Kaczmarz algorithms might diverge in the absence of the persistence of excitation [8]. 


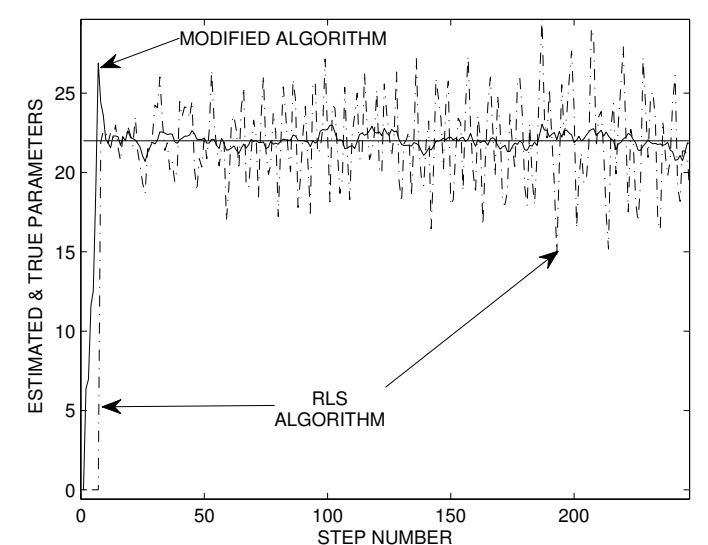

FIG 2. System (4), (7) with $q=1,2,3, \sigma^{2}=1$, was simulated with modified Kaczmarz algorithm (20),(21) plotted with a solid line and RLS algorithm (30),(31) plotted with a dash-dot line. An equal perturbation term was added to the right hand side of the equations for matrix $\Gamma_{k}$. True value of the parameter is equal to 22 .

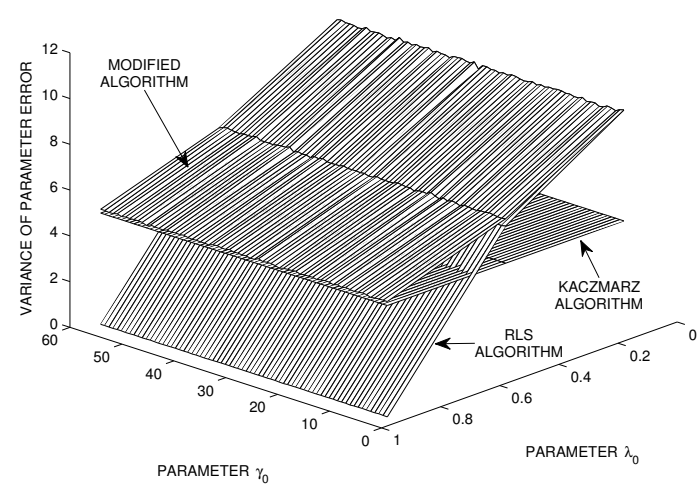

FIG 3. A variance of the parameter mismatch $E\left\|\tilde{\theta}_{k}\right\|^{2}$ which is estimated at steady-state via a sum of sample variances for a sufficiently large sample size. System (4), (7) with $q=1,2,3, \quad \sigma^{2}=3$ was simulated with Kaczmarz algorithm (20) with $\Gamma_{k}=I$, modified Kaczmarz algorithm (20),(21), and RLS algorithm (30),(31). The variance is plotted as a function of two parameters forgetting factor $\lambda_{0}$ and initial value $\gamma_{0}$ with $\lambda_{1}=1$.

\section{EXAMPLE: A FREQUENCY DOMAIN IDENTIFICATION METHOD OF DC MOTOR}

DC motors are widely used as actuators in control systems to provide a rotary motion to a variety of electromechanical devices and servo systems. In order to achieve a high performance regulation the parameters of the motor should be identified. Newton's law combined with Kirchhoff's law yield to the following transfer function of DC motor, where the angle is the output and the voltage is the input:

$$
H(p)=\frac{K}{p\left(T_{1} p+1\right)\left(T_{2} p+1\right)}
$$

where $T_{1}=0.1[\mathrm{sec}]$ and $T_{2}=0.02[\mathrm{sec}]$ are mechanical and electrical time constants respectively and $K=$ $100\left[\frac{1}{s e c}\right]$ is a gain.

First, true values of the magnitude and the phase of the transfer function $H(i q)$ are calculated

$$
\begin{aligned}
|H(i q)| & =\frac{K}{q \sqrt{1+q^{2} T_{1}^{2}} \sqrt{1+q^{2} T_{2}^{2}}} \\
\psi_{q *} & =-90^{\circ}-\arctan \frac{q\left(T_{1}+T_{2}\right)}{1-q^{2} T_{1} T_{2}}
\end{aligned}
$$

for the following set of frequencies $q=3,7,15,25,45,100,300$ $\left[\frac{1}{s e c}\right]$. Alternatively, the magnitude and the phase of the response can be calculated using the MATLAB function bode.

Secondly, a multi-frequency input sequence is specified in a form of (1) and the model for the output is specified in a form of (7), where the parameters are updated via algorithm (20) and (21). A variance of the measurement noise is $\sigma^{2}=0.05$. Finally, the magnitude and the phase of the response are calculated in MATLAB using (13) and (14). Frequency responses for plant (32) are summarized in Table 1, where true values of the magnitude $|H(i q)|$ and the phase $\psi_{q *}$ calculated via (33), (34) are compared to the values of the magnitude $|\hat{H}(i q)|=\frac{B_{q k}}{A_{q}}$ and the phase $\psi_{q k}$ estimated via average values at steadystate for 60000 points using algorithm (20), (21). Table 1 shows very good agreement between estimated and true values of the magnitude and phase. Estimated values of the time constants and the gain for plant (32) can be calculated using frequency response data listed in Table 1. Since the number of measured points is larger than the number of unknown parameters (two time constants and one gain) the estimated values of these parameters are over-determined and can be found using a least-squares method.

\begin{tabular}{|l|l|l|l|l|l|}
\hline$q$, sec $^{-1}$ & 0 & 3 & 7 & 15 & 25 \\
\hline$|H(i q)|$ & $\infty$ & 31.87 & 11.59 & 3.54 & 1.33 \\
\hline$|\hat{H}(i q)|$ & $\infty$ & 31.88 & 11.6 & 3.54 & 1.33 \\
\hline$\psi_{q *}$, Deg & -90 & -110.13 & -132.96 & -163.01 & -184.76 \\
\hline$\psi_{q k}$, Deg & -90 & -110.12 & -132.92 & -162.92 & -184.63 \\
\hline \hline$q$, sec $^{-1}$ & 45 & 100 & 150 & $\infty$ \\
\hline$|H(i q)|$ & 0.3583 & 0.0445 & 0.014 & 0 \\
\hline$|\hat{H}(i q)|$ & 0.3581 & 0.0449 & 0.0144 & 0 \\
\hline$\psi_{q *}$, Deg & -209.46 & -237.72 & -247.75 & -270 \\
\hline$\psi_{q k}$, Deg & -209.2 & -237.36 & -246.29 & -270 \\
\hline \multicolumn{7}{|l}{ Table 1. Frequency Response } \\
\hline
\end{tabular}

Table 1. Frequency Response

A transient performance of algorithm (20) and (21) is illustrated in Figure 4 and Figure 5.

\section{DISCUSSION}

The methods which are able to solve rapidly the frequency response identification problems are very demanded in such application areas as communication, instrumentation, civil engineering, biomedicine and others. 


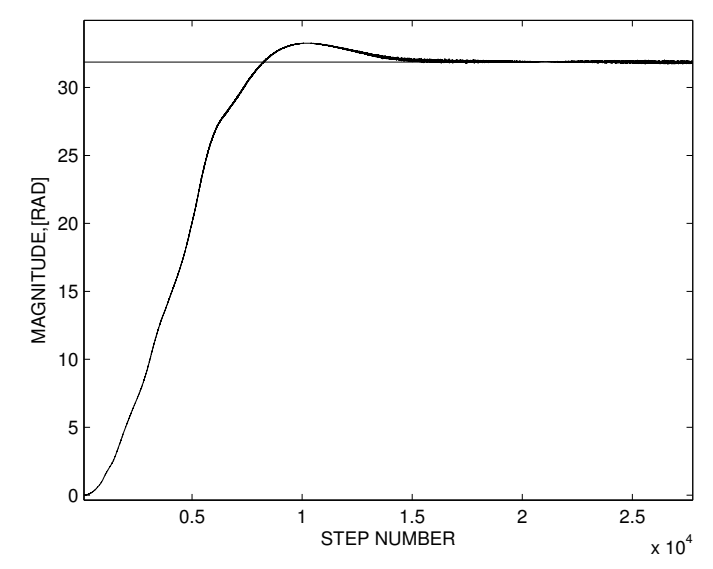

FIG 4. The values of the magnitude $|\hat{H}(i q)|=\frac{B_{q k}}{A_{q}}$ estimated via modified algorithm (20), (21) are evaluated at frequency $q=3\left[\frac{1}{s e c}\right]$ and plotted as a function of a step number. A true value of the magnitude $|H(i q)|=\frac{B_{q *}}{A_{q}}$ calculated via (33) is equal to 31.87 .

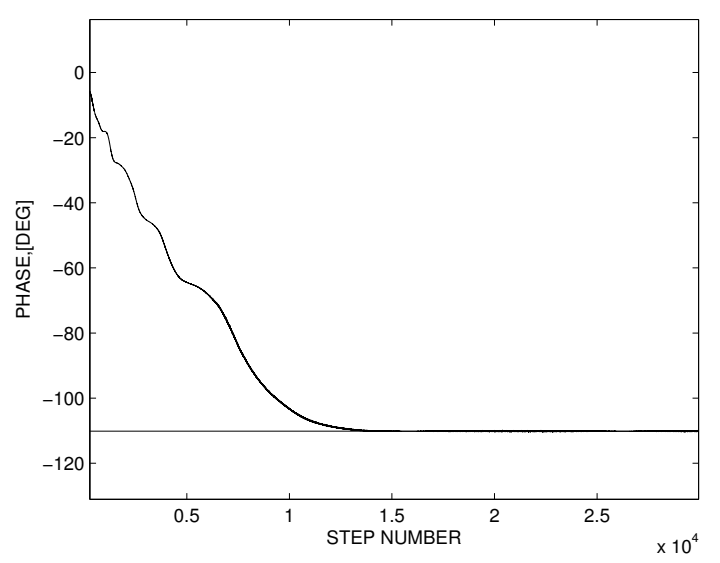

FIG 5. The values of the phase $\psi_{q k}=-\arctan \frac{b_{q k}}{a_{q k}}$ estimated via modified algorithm (20), (21) are evaluated at frequency $q=3\left[\frac{1}{s e c}\right]$ and plotted as a function of a step number. A true value of the phase $\psi_{q *}$ calculated via (34) is equal to $-110.13^{0}$.

The performance of the transmission channels and cables is identified in communications by sending a test signal and recording the frequency response.

Frequency response curves of microphones, amplifiers, loudspeakers, CD players and other devices can be identified in a similar way in the instrumentation area.

Frequency response functions of such civil engineering structures as buildings, towers, bridges and others are used for active vibration mitigation and reduction of damaging effects of such dynamic loads as high winds, extreme waves and strong earthquakes [5].

Besides, a frequency domain analysis can also be used in biomedical signal processing applications for rapid decom- position of rhythmic oscillations of the blood pressure and heart rate into different frequency components and for quantification of the power of these oscillations at each specific frequency [10].

Finally, radar/sonar and seismic signal processing applications should also be mentioned in a frequency domain context.

Simple, fast and robust algorithms are required for estimation of the frequency components of multi-frequency output measurements in these and many other applications. The performance of Kaczmarz algorithm is improved in this paper which makes the Kaczmarz projection method applicable to many problems mentioned above.

\section{REFERENCES}

[1] Astrom K. J., Wittenmark B., Adaptive Control, Addison-Wesley, 1989.

[2] Avedyan E.D., Modified Kaczmarz Algorithms for Estimating the Parameters of Linear Plants, Autom. Remote Control, vol. 39, N 5, 1978, pp. 674-680.

[3] Goodwin G. and Sin K., Adaptive Filtering, Prediction and Control. Englewood Cliffs, NJ: Prentice-Hall, 1984.

[4] Fomin, V. N., Fradkov A.L. and Yakubovich V.A. Adaptive Control of the Dynamic Plants, Nauka, Moscow, 1981, (in Russian).

[5] Jin G., Sain M., and Spencer B., Frequency Domain System Identification for Controlled Civil Engineering Structures, IEEE Transactions on Control Systems Technology, vol. 13, N 6, Nov. 2005, pp. 1055-1062.

[6] Horn R. and Johnson C. Matrix Analysis, Cambridge University Press, 1985.

[7] Landau I. and Zito G., Digital Control Systems: Design, Identification and Implementation, SpringerVerlag, London, 2006.

[8] Lefeber E. and Polderman J. On the Possible Divergence of the Projection Algorithm, IEEE Transactions on Automatic Control, vol. 40, N 3, March 1995, pp. 495-497.

[9] Ljung L. System Identification: Theory for the User, Prentice Hall, 1999.

[10] Parati G., Saul J., Di Rienzo M., Giuseppe Mancia G., Spectral Analysis of Blood Pressure and Heart Rate Variability in Evaluating Cardiovascular Regulation, Hypertension vol. 25, 1995, pp. 1276-1286, American Heart Association Inc.

[11] Stotsky A. Automotive Engines: Control, Estimation, Statistical Detection, Springer-Verlag, BerlinHeidelberg, 2009.

[12] Stotsky A. Recursive Trigonometric Interpolation Algorithms, Proceedings of the Institution of Mechanical Engineers, Part I: Journal of Systems and Control Engineering, Volume 224, Number 1, 2010, pp. 65-77.

[13] Stotsky A. A New Frequency Domain System Identification Method, Proceedings of the Institution of Mechanical Engineers, Part I: Journal of Systems and Control Engineering, 2011, to appear.

[14] Vandoorn T., De Belie F., Vyncke T., Melkebeek J. and Lataire P., Generation of Multisinusoidal Test Signals for the Identification of Synchronous-Machine Parameters by Using a Voltage-Source Inverter, IEEE Transactions on Industrial Electronics, vol. 57, N 1, Jan. 2010, pp. 430 - 439. 